\title{
Instruction Folding in a Hardware-Translation Based Java Virtual Machine
}

\author{
Hitoshi Oi \\ Department of Computer Science \\ The University of Aizu \\ Aizu-Wakamatsu, JAPAN
}

\begin{abstract}
Bytecode hardware-translation improves the performance of a Java Virtual Machine (JVM) with small hardware resource and complexity overhead. Instruction folding is a technique to further improve the performance of a JVM by reducing the redundancy in its stack-based operations. However, the variable instruction length of the Java bytecode makes the folding logic complex. In this paper, we propose a folding scheme with reduced hardware complexity and evaluate its performance. For eleven benchmark cases, the proposed scheme folded $7.1 \%$ to $36.8 \%$ of the bytecodes which correspond to $74.0 \%$ to $99.7 \%$ of the PicoJava-II's folding performance.
\end{abstract}

\section{Introduction}

In this section, we present an introduction to the hardware-translation based Java Virtual Machine and the instruction folding.

\subsection{Hardware-Translation of Java Bytecode}

Hardware-translation is a technique to enhance the performance of the Java Virtual Machine (JVM) [1] by dynamically replacing the bytecodes to native machine instructions ${ }^{1}$. A small translation logic is inserted between the fetch and decode stages of the processor pipeline. When a flag in the processor's status register indicates that the fetched instruction is a Java bytecode, it is converted into native instructions by the translation unit. If the native instruction is fetched, it bypasses the translation logic. In theory, the decode and later stages of the processor pipeline do not see the difference between the native and Java bytecode execution modes which implies that the changes to the processor core is kept minimum.

Table 1 shows an example of the bytecode translation. In this example, two local variables which are assigned local variable indexes 3 and 4, are added and the result is written to the local variable 3. First two bytecodes, ILOAD_3 and ILOAD_4 push the values of two local variables onto the stack. Following the ARM Jazelle's specification, R0 to R3 are used to hold the top four words

1. In the court order [2] dated September 30, 2003, ARM's Jazelle and Nazomi's U.S. Patent No. 6,332,215 are distinguished as follows. While Nazomi's patent translates Java bytecodes into native instructions before reaching the decode stage of the CPU, ARM's Jazelle translates bytecodes into controls signals. While this difference may be important for the patent issues, it is not essential for the ideas discussed in this paper. Therefore, the readers of this paper can interchangeably read "native instructions" as "the sequence of control signals corresponding to the native instructions", vice versa. 
of the operand stack in this example ${ }^{2}$. Therefore, the first two bytecodes are translated into two load word instructions (LDR) using R7 which holds the address of the local variable 0 and corresponding offsets. Next bytecode, IADD, pops and adds two top of stack words and pushes the result onto the stack. This bytecode is translated into a native instruction which adds two registers R0 and R1. The last bytecode, ISTORE_3 pops the top of stack word and writes it to the local variable 3 . This bytecode is replaced with a store word instruction (STR).

\begin{tabular}{|l|l|}
\hline Bytecode & ARM Instruction \\
\hline ILOAD_3 & LDR R0, [R7, \#12] \\
ILOAD_4 & LDR R1 [R7, \#16] \\
IADD & ADD R0, R1 \\
ISTORE_3 & STR R0, [R7, \#12] \\
\hline
\end{tabular}

Table 1: An example of bytecode hardware-translation.

As shown in the above example, the translation unit reads a single bytecode at a time and generates a short sequence of native machine instruction(s). The hardware-translation is limited to the simple 134 bytecodes such as load, store, and arithmetic/logical operations on the stack [3]. Complex bytecodes, such as new (create a new object), are emulated by the software. By limiting the complexity of the translation mechanism, the hardware resource overhead and the performance gain are balanced: in the case of Jazelle, it is reported that $8 \mathrm{x}$ performance gain was achieved by 12K gates, while typical dedicated or co-processors for JVMs are around 20-25K [4].

\subsection{Instruction Folding of Java Bytecode}

As shown in the bytecode sequence in Table 1, there is inherent redundancy in the Java bytecode which comes from its stack architecture. In the above example, it took four bytecodes to add two variables and write the result back to one of them. Almost all microprocessors can do an equivalent operation with a single instruction, such as $A D D A, B$. This technique of merging multiple bytecodes into a single instruction is called the instruction folding and can be found in Java processors [8]. However, there are two issues when applying the instruction folding scheme to the hardware-translation based JVMs. First, most embedded microprocessors, which are the target platform of the hardware-translation JVM, are RISC architectures. This implies that arithmetic and logic operations cannot take memory locations as operands. If the part of operand stack for the local variables is allocated on the main memory (which is likely as shown in the example in Table 1), the instruction folding is not possible. Previously, we proposed to add a small register file to the datapath of the JVM to reduce the number of memory accesses caused by the local variables [7]. This extra register file (called local variable cache in [7]) also makes instruction folding possible on the hardware-translation based JVMs.

Another issue is the hardware complexity of the logic circuit that detects foldable bytecode sequences. In Sun's PicoJava-II, up to four bytecodes are folded into a single microprocessor operation, meaning four bytecodes are decoded simultaneously. Compared to the single bytecode

2. Since the number of registers assigned to hold operand stack entries is fixed to four, they are considered to be used as a circular buffer with a modulo 4 pointer to R0 to R3 [3]. If more than four items are pushed onto the stack, spill and restore operations are required. For the sake of simplicity, however, these operations are omitted in this example. 
decoding policy of the hardware translation, decoding four bytecodes for folding detection may be too complex. Moreover, the variable length of Java bytecode adds more complexity to the detection of the foldable sequences. The first bytecode opcode is uniquely pointed to by the program counter (PC), but the next bytecode could be either at $P C+1,2$ or even at $P C+3$ depending on the length of the first bytecode (excluding non-foldable bytecodes). This means that to obtain the $i$-th opcode, where $i=2 \cdots 4$, we have to decode $1 \cdots i-1$ bytecodes beforehand.

In this paper, we present an instruction folding mechanism that provides similar performance to that of PicoJava-II with a reduced hardware complexity. The proposed scheme is evaluated by bytecode level simulations and analysis of bytecode sequence patterns that contribute to instruction folding is presented.

The rest of this paper is organized as follows. In the next section, an overview of PicoJava-II's folding scheme is presented. In Section 3, we propose an instruction folding scheme that alleviates the hardware complexity of PicoJava-II's scheme. In Section 4, the experimental environment including Java Virtual Machine and benchmark programs are first described, and then the proposed schemed is evaluated by comparing it to the performance of the PicoJava-II through simulations. Related work and conclusions are presented in Sections 5 and 6, respectively.

\section{PicoJava-II's Instruction Folding Scheme}

In this section, the instruction folding scheme of Sun's PicoJava-II and its source of hardware complexity are described. In PicoJava-II, Java bytecodes are classified into six types [8]:

LV: A local variable load or load from global register or push constant (e. g. ILOAD)

OP: An operation that uses the top two entries of stack and that produces a one-word result (IADD)

BG2: An operation that uses the top two entries of the stack and breaks the group (IF ICMPEQ)

BG1: An operation that uses only the topmost entry of the stack and breaks the group (IFEQ)

MEM: A local variable store, global register store, and memory load (ISTORE)

NF: A nonfoldable instruction (GOTO)

Based on this classification, the following nine bytecode sequences (groups) are defined:

\section{Group 1 LV LV OP MEM}

Group 2 LV LV OP

Group 3 LV LV BG2

Group 4 LV OP MEM

\section{Group 5 LV BG2}

Group 6 LV BG1

\section{Group 7 LV OP}

\section{Group 8 LV MEM}




\section{Group 9 OP MEM}

Figure 1 shows the block diagram of the PicoJava-II's foldable sequence detection circuit. Note that this diagram is reproduced from [8] with the following changes. First, while PicoJava-II has extended two-byte opcode instructions, they are specific to PicoJava-II's implementation and unrelated to other JVMs in general, including ours. Therefore, the folding type decoders handle only single byte opcodes. Second, in PicoJava-II, each instruction byte is associated with the length of the bytecode by assuming that the byte is the opcode. This instruction length is decoded in the instruction cache which is in the fetch stage of the pipeline. In this paper, we assume that the hardware translation module of the Java bytecode is inserted between the fetch and decode stages and try to minimize the changes to the processor core. Therefore, unlike PicoJava-II, decoding of the instruction length is also performed in the hardware translation module.

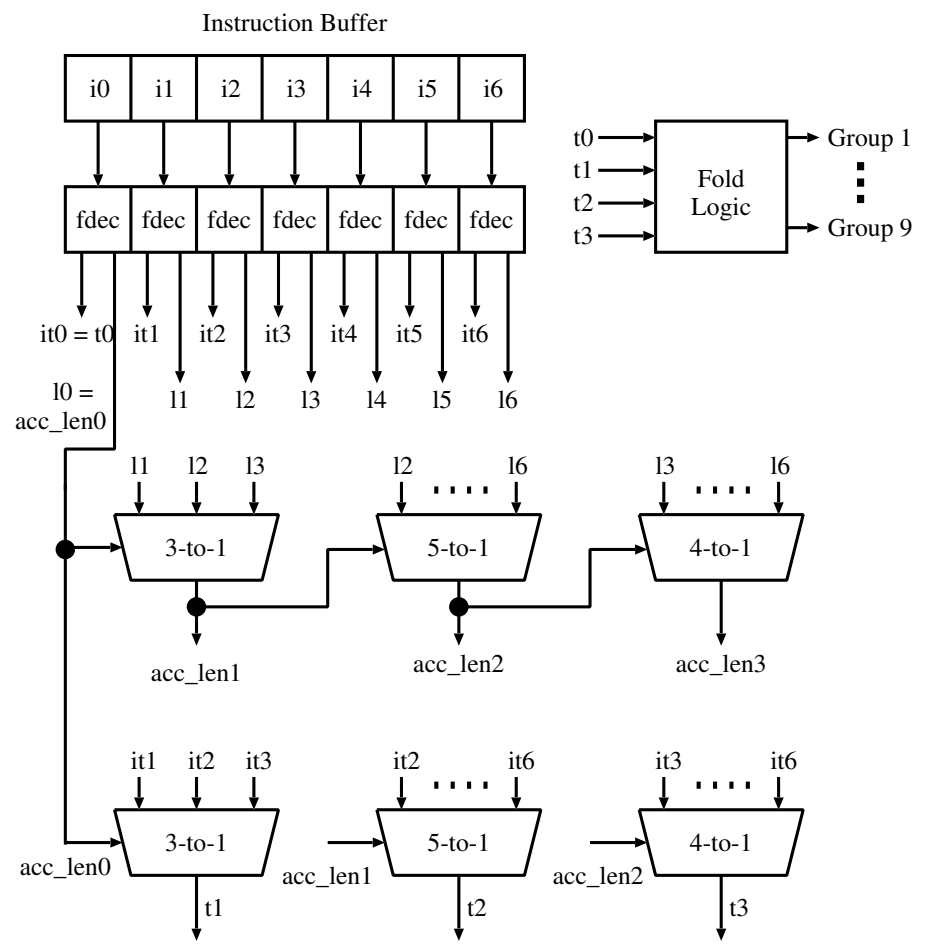

Figure 1: PicoJava-II's foldable bytecode detection logic.

In the instruction buffer, there are seven entries and each entry consists of an instruction byte (i0 to i6). All instruction bytes are speculatively decoded by folding type decoders (fdec) and generate instruction types (it0 to it6) and instruction lengths (10 to 16). The first byte in the buffer (i0) is always the opcode of the first bytecode (b0). Therefore, it0 is actually the folding type of the first bytecode ( $\mathrm{t} 0$ ).

Since the length of foldable bytecodes varies from one (e. g. IADD) to three (e. g. SIPUSH), the length of the first bytecode (10) is given to a 3-to-1 MUX to select the folding type of the second bytecode ( $\mathrm{t} 1$ ) from it 1 to it 3 . The length of the first bytecode (10) is also used to select the 
accumulated length of the first and the second bytecodes (acc len1) from 11 to 13, which in turn selects the folding type of the third bytecode ( $\mathrm{t} 2$ ) from it 2 to it6 as well as to select the accumulated length of the first through third bytecodes (acc_len2).

Similarly, acc_len 2 selects the folding type of the fourth bytecode (t3) from it 3 to it 6 and the accumulated length of the first through fourth bytecodes (acc len3).

The folding detection logic takes folding types of all four bytecodes (t0 to t3) and enables one of nine output (Group 1 to Group 9) if any foldable sequences are detected. Since a longer sequence has a priority, for example, for an LV LV OP MEM sequence, only Group 1 output is enabled (i. e. Group 2 output is disabled).

Note that, the length of the first bytecode (10) is propagated through three multiplexers (MUXes) to determine the folding type of the fourth bytecode ( $\mathrm{t} 3)$. $\mathrm{t} 3$ is then fed into the folding detection logic and then finally a possible folding sequence is determined.

It has been pointed out that the instruction folding unit (IFU) can be a critical path in the decode stage of the PicoJava-II processor pipeline [9]. Together with the fact that the hardware-translation is performed on a single bytecode basis, we consider it is worth re-exploring the design space of the instruction folding logic by taking the hardware overhead and complexity into account.

\section{Three Bytecode Folding Scheme with Reduced Complexity}

In this section, we propose an instruction folding scheme that takes up to three bytecodes with reduced hardware complexity and still provides a similar performance to that of PicoJava-II. The primary source of complexity in the PicoJava-II's folding mechanism is the variable length of the bytecode, especially, the length of the LV type bytecodes that varies from one to three bytes. To reduce this complexity, we modified the PicoJava-II's scheme in the following two points. First, we limit the number of folding bytecodes to three (i. e. Group 1 is excluded). Next, we exclude SIPUSH, which is the only three byte long LV type bytecode and handle it as an NF bytecode.

As we will see in the next section, in general, the fraction of Group 1 sequence (LV LV OP MEM) is small and the instruction count of SIPUSH is also small compared to other LV bytecodes. The lengths of MEM, BG1 and BG2 are also variable. However, these bytecodes are always at the end of the foldable sequence and hence do not affect the position of the opcodes of other bytecodes in a foldable sequence.

Figure 2 shows the block diagram of the proposed folding scheme. While the instruction buffer still stores seven instruction bytes, it only (speculatively) decodes the folding types (it0 to it4) and lengths (10 to 14) of the first five bytes (i0 to i4) since the opcode of the third bytecode in foldable sequences does not go beyond i4. The folding type of the second bytecode ( $\mathrm{t} 1$ ) is selected from either it 1 or it 2 based on the length of the first bytecode (acc_len0) which is actually 10. The accumulated instruction lengths up to second and third bytecodes (acc Jen1 and acc len12, respectively) are obtained in the same manner as in the PicoJava-II with fewer candidates.

The hardware complexity of the proposed mechanism is reduced in the following points. First, since we have dropped Group 1, the length of the multiplexer chain to obtain the accumulated instruction lengths has reduced from three to two. Second, the sizes of the MUXes for $\mathrm{t} 1$ and $\mathrm{t} 2$ as well as acc_len1 and acc_len2 have changed from 3-to-1 and 5-to-1 to 2-to-1 and 3-to-1, respectively. Moreover, the number of folding type decoders (fdec) has been reduced from seven to five.

Block diagrams in Figures 1 and 2 do not include the circuit which handles the cases where the bytecode sequence is foldable but its length exceeds the size of the instruction buffer. In such a 
Instruction Buffer
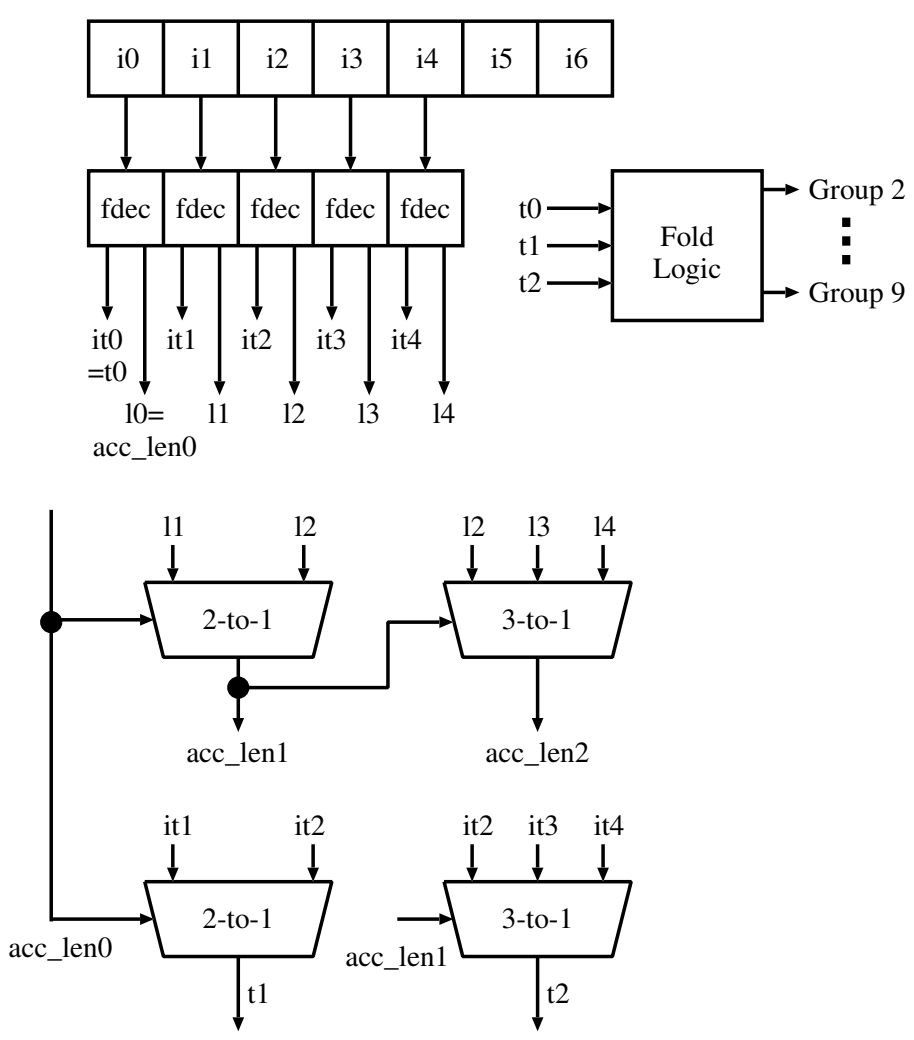

Figure 2: Proposed foldable bytecode detection logic.

case, the folding type of the corresponding bytecode must be changed to NF regardless of its predecoded folding type. The proposed scheme is also simpler than PicoJava-II in this part because only acc_len $0=1$ or 2 and acc_len $1=2$ to 4 are valid for the folding type signals $t 1$ and $t 2$, respectively. Therefore, all the instruction bytes for a foldable sequence are always in the instruction buffer in the propose mechanism.

A complete and precise estimation of the hardware overhead and speed cannot be obtained without (at least) the datapath that is controlled by the folding pattern signals detected in the pattern detection module. At present, we do not have such a complete model of a hardware-translation based JVM and also the emphasis of this paper is placed on the optimization of the folding sequences. However, as a metrics of the hardware complexity and operation speed, we wrote Verilog models for the circuits in Figures 1 and 2 and synthesized them under the $0.35 \mu$ rule. In PicoJava-II's model (Figure 1), the delay of the longest path (from i0 to Group 1) was 2.82ns. On the other hand, in the proposed scheme (Figure 2), the delay of the longest path (from i0 to Group 2) was 2.50ns, which is a reduction of $11 \%$. We also compared the logic circuit areas and it was found that the proposed scheme occupied 35\% less area than that of PicoJava-II. 


\section{Performance Evaluation}

In this section, we evaluate the performance of the proposed folding mechanism and compare it to that of PicoJava-II. We also use the two bytecode version of PicoJava-II's mechanism which only works for Groups 5 to 9 as another reference. First, we present the simulation environment including JVM and benchmark programs and the simulation results follow.

\subsection{Experimental Environment}

For the JVM and Java Runtime Environment, we use Kaffe version 1.0.7 [10]. Kaffe is an opensource implementation of the JVM and we compiled it with "-with-engine=intrp" option so that all bytecodes are interpreted. It is assumed that a 16-entry local variable cache is attached to the JVM which works as described in [7]. This size of the local variable cache can accommodate all local variables of DES, ECM and PNG, and is effectively large enough for SAXON with XSLTMark test case documents and GrinderBench.

Note that, the LV type bytecodes can be further divided into two classes. The first class is those actually accessing local variables, such as ILOAD. If the local variable cache does not have a valid copy of the accessed local variable, it must be loaded from the memory. Therefore, for example, a Group 7 sequence ILOAD_1, IADD, is effectively not folded if the local variable 1 is not present in the local variable cache. Another class of LV bytecodes is those not actually accessing local variables, such as ICONST_0. When such a bytecode appears in any foldable sequence, it is always folded.

The benchmark programs used for the evaluation are listed in Table 2. The first set of benchmark programs is SAXON Version 6.3, an XSLT processor [11], driven by four test case XML documents from XSLTMark [12]. We chose four test case documents, chart, decoy, encrypt and trend, based on the average number of bytecode executed for a method invocation and the functional categories defined in the XSLTMark.

The Embedded CaffeineMark consists of five tests, Sieve, Loop, Logic, Method and Float [13]. Each of these tests is basic and tries to measure various aspects of JVM. Composite results of all five tests are used.

DES is a DES based encryption and decryption of a text file using the Bouncy Castle Crypto Package [14]. A text file of $5 \mathrm{~KB}$ is first encrypted and then decrypted using the sample program included in the Bouncy Castle Crypto package

(src/org/bouncycastle/crypto/examples/DESExample.java).

PNG extracts properties of a PNG image file $(512 \times 512$ from [16] $)$ such as pixel size and bit depth using com.sixlegs.png PNG decoder and its sample program

PropertiesExample. java [15].

Next four benchmark programs, chess, crypto, kxml and pngdecode are from GrinderBench [5]. It is a suite of Java kernels developed by the Embedded Microprocessor Benchmark Consortium (EEMBC) [6] and focuses on the Connected Limited Device Configuration (CLDC) part of the Java 2 Micro Edition (J2ME).

The fraction of each bytecode type and the average run length for each benchmark program are presented in Tables 3. The average run length is the number of contiguously executed bytecodes without interruption by invocation or return. The higher this number, the more chances of folding. The number in parentheses in the LV column indicates the fraction of three-byte LV bytecode (SI- 


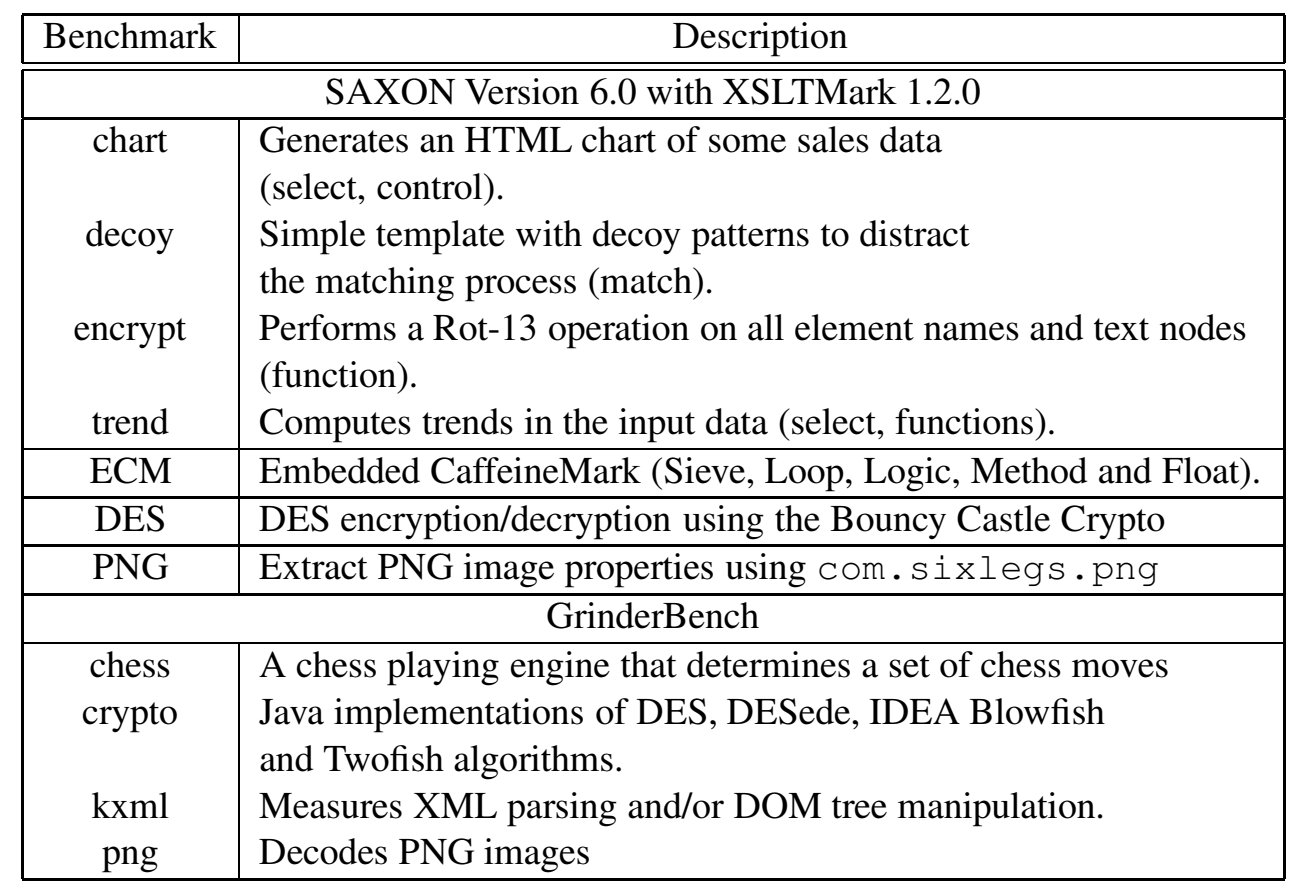

Table 2: Benchmark program description.

PUSH). Since this bytecode is handled as an NF in the proposed scheme, this number indicates the cases where PicoJava-II can fold instructions but the proposed scheme cannot.

\subsection{Simulation Results}

In this section, we present and analyze the results of simulations. Figure 3 shows the breakdown of the folded bytecodes for SAXON with four XSLTMark test cases. Compared to other benchmark programs, the fractions of folded bytecodes for SAXON are small. Two reasons can be found in Tables 3. First, they have high fractions of non-foldable bytecode (NF) ranging from $26.8 \%$ to $40.3 \%$. Second, the average run lengths are short, leading to fewer chances of folding. Among these four cases, the highest folding performance is archived in chart. With the proposed scheme, $18.0 \%$ of bytecodes are folded, which is $96 \%$ of PicoJava-II (18.8\%) as shown in Table 4. While the difference is small, Groups 1, 4 and 7 are major contributors to it.

The performance of the proposed scheme is almost the same as PicoJava-II's for other three test cases (98.3, 98.8 and 98.5\%). The proposed scheme cannot fold Group 1, but more Groups 2 and 9 are folded in the proposed scheme. The two bytecode version of PicoJava-II's scheme (PJ2B) performs significantly worse than the other two schemes (68.6\% to $81.9 \%$ of PicoJava-II). In PJ2B, three and four byte sequences (Group 1 to 4 ) are partially detected as two byte sequences and folded. The increases resulting from partially folded sequences are mostly in Group 5 and 7. The most evident case is Group 5 in chart in which PJ2B folds $4.6 \%$ more bytecodes than the original PicoJava-II. Decoy has a shorter run length and a higher fraction of NF bytecode than those of encrypt. However, all three folding schemes perform better on decoy than on encrypt. 


\begin{tabular}{|c|c|c|c|c|c|c|c|}
\hline \multirow{2}{*}{$\begin{array}{l}\text { Bench } \\
\text {-mark }\end{array}$} & \multicolumn{6}{|c|}{ Bytecode Types (\%) } & \multirow{2}{*}{$\begin{array}{c}\text { Run } \\
\text { Length }\end{array}$} \\
\hline & LV & OP & BG1 & BG2 & MEM & $\mathrm{NF}$ & \\
\hline \multicolumn{8}{|c|}{ SAXON with XSLTMark } \\
\hline chart & $44.4(0.7)$ & 4.3 & 7.7 & 12.6 & 4.1 & 26.8 & 11.6 \\
\hline decoy & $44.4(0.3)$ & 2.3 & 8.3 & 9.9 & 4.0 & 31.1 & 8.8 \\
\hline encrypt & $42.5(0.1)$ & 4.0 & 7.4 & 14.0 & 3.3 & 28.8 & 10.8 \\
\hline trend & $39.9(0.1)$ & 1.6 & 9.8 & 5.8 & 2.6 & 40.3 & 4.9 \\
\hline ECM & $45.3(0.0)$ & 5.3 & 9.8 & 13.8 & 7.1 & 18.9 & 69.0 \\
\hline$\overline{\mathrm{DES}}$ & $43.9(0.7)$ & 24.5 & 1.7 & 10.1 & 9.2 & 10.7 & 63.3 \\
\hline PNG & $42.8(2.5)$ & 11.0 & 3.8 & 13.3 & 2.9 & 26.3 & 24.3 \\
\hline \multicolumn{8}{|c|}{ GrinderBench } \\
\hline chess & $44.1(0.0)$ & 6.1 & 4.5 & 16.5 & 3.5 & 25.4 & 33.6 \\
\hline crypto & $45.6(2.0)$ & 21.8 & 2.4 & 9.5 & 11.0 & 9.7 & 35.0 \\
\hline $\mathrm{kxml}$ & $43.3(0.6)$ & 3.6 & 8.8 & 12.4 & 2.7 & 29.3 & 10.8 \\
\hline pngdecode & $39.6(1.1)$ & 9.6 & 10.6 & 5.9 & 9.1 & 25.1 & 14.2 \\
\hline
\end{tabular}

Table 3: Benchmark program bytecode analysis. The numbers in parentheses in the LV column are the fractions of three byte LV bytecode (i. e. SIPUSH). The right-most column (Run Length) shows the average number of contiguously executed bytecodes without interruption by invocation or return.

Figure 4 shows the breakdown of the folded bytecodes in the Embedded CaffeineMark, DES and PNG. The average run length of Embedded CaffeineMark (ECM) is 69.0 bytecodes, which is the longest among the benchmark programs used in this paper. This long sequence of uninterrupted bytecodes leads to high folding ratios $(24.0 \%$ to $29.8 \%)$. One thing that should be noted is that, while $7.1 \%$ of bytecodes are of MEM type, we hardly see Group 4 in PicoJava-II and the proposed scheme. Instead, $3.1 \%$ of bytecodes are folded in Group 8 in all three schemes. Therefore, a large fraction of MEM bytecodes follow LVs: meaning either they copy one LV to another (e. g. ILOAD_1 and ISTORE_2) or initialize an LV by a constant (ICONST 2 and ISTORE_3). 1.6\% of bytecodes are folded in Group 1 in PicoJava-II. However, when it is compared to the sums of Groups 1 and 2 in the proposed scheme, the difference shrinks to $0.5 \%$. This implies that the increase of Group 2 in the proposed scheme effectively absorbs most of the Group 1 sequences. The proposed scheme achieves a relative performance of $98.1 \%$ of PicoJava-II's scheme. In PJ2B, we see that the fraction of Group 7 is significantly higher than PicoJava-II and the proposed scheme (3.6\% against $0.4 \%$ ). This is possibly because the LV OP part of Groups 1 and 2 were detected and folded as Group 7 in PJ2B. The relative performance of $\mathrm{PJ} 2 \mathrm{~B}$ was $80.6 \%$.

DES has a very long average run length (63.3, next to ECM) and the lowest fraction of NF bytecodes $(10.7 \%)$. It also has a very high fraction of OP bytecodes $(24.5 \%)$, which leads to large numbers of folded bytecodes in Groups 2, 7 and 9. These two properties confirm the computationintensive nature of DES encryption/decryption algorithms. While the fraction of MEM bytecodes is the second highest in the benchmarks used, the fraction of Group 8 foldings is quite low $(0.1 \%)$. This means, unlike ECM, MEM bytecodes are used to store the results of OP bytecodes rather than 


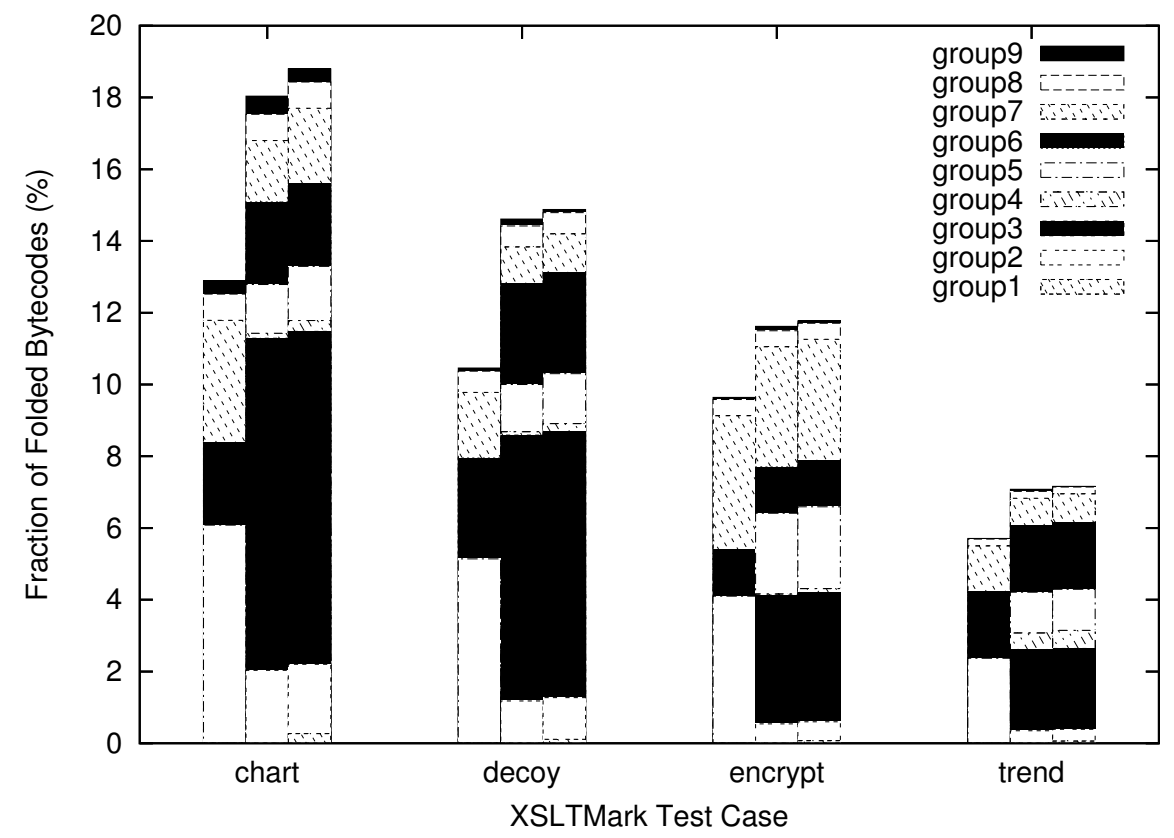

Figure 3: Breakdown of the folded bytecodes for SAXON with XSLTMark test cases. For each benchmark, three bars indicate Two-Bytecode version of PicoJava-II, proposed scheme, PicoJava-II's scheme (left to right).

initialization or copy of local variables. In DES, the fractions of three-byte LV bytecodes (SIPUSH) is relatively high $(0.7 \%)$ and the fraction of Group 1 is also high among the benchmark programs used. Since SIPUSH and Group 1 are excluded in the proposed scheme, these two properties are disadvantages for the proposed scheme. The fraction of Group 2 in the proposed scheme is not high enough to cover the Group 1 sequences which are folded in PicoJava-II and also the fraction of Group 7 is slightly lower in the proposed scheme. The proposed scheme performed $95.2 \%$ of PicoJava-II's folding scheme. In PJ2B, we see that the bar for Group 7 is much longer than in PicoJava-II or in the proposed scheme, but it is not long enough to cover Groups 1 to 4 that are missing in $\mathrm{PJ} 2 \mathrm{~B}$. The relative performance of $\mathrm{PJ} 2 \mathrm{~B}$ is only $67.1 \%$ of the original four-byte folding scheme.

PNG has a relative short run length and a high NF bytecode fraction. These characteristics make the folding performance in PNG similar to those in SAXON. Its fraction of NF bytecode is $26.3 \%$ and the average run length is only 24.3 bytecodes. As a result, even with the PicoJava-II's scheme, only $17.7 \%$ of bytecodes are folded. Also, $2.9 \%$ of bytecodes are folded as Group 1 in PicoJava-II and $2.5 \%$ of bytecodes are SIPUSH, both of which are disadvantages for the proposed scheme. The relative performance of the proposed scheme is $88.2 \%$. With PJ2B, the fractions of Groups 5 and 7 are increased significantly by partially folding the longer sequences (Groups 1 to 4). Its relative performance is $77.2 \%$. 


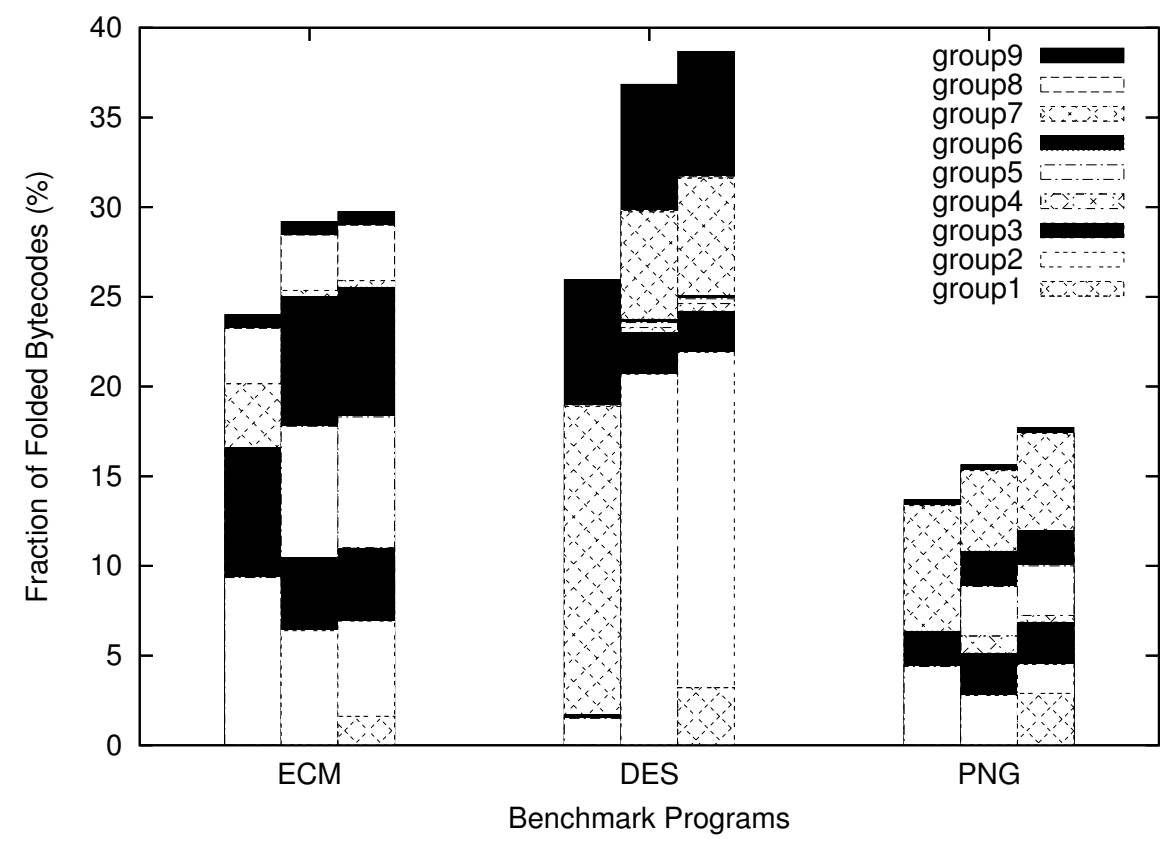

Figure 4: Embedded CaffeineMark, DES, PNG. For each benchmark, results of Two-Bytecode version of PicoJava-II, proposed scheme, PicoJava-II's scheme are shown from left to right.

GrinderBench is a collection of Java applications for embedded platforms and it examines different aspects JVMs. The behavior of crypto, kxml and pngdecode have some similarities with other benchmark programs used in this paper: DES, SAXON and PNG, respectively. However, the differences in their run time behaviors look to be more emphasized. Consequently, they also show quite different folding performance (Figure 5).

Chess has a relative long run length. The difference between the performance of the proposed scheme and that of PicoJava-II is quite small. The relative performance of the proposed scheme is $99.7 \%$, which is the best among the benchmark progrms used in this paper.

On the contrary, the difference between folding schemes is stressed in crypto. It has the lowest fraction of NF bytecode (9.7\%) and a moderate run length (35.0 bytecodes). These factors contribute to the good folding performance of PicoJava-II (38.1\%). However, $2.0 \%$ of bytecodes are SIPUSH and $8.4 \%$ of bytecodes are folded as Group 1 . These two cases cannot be handled by the proposed scheme and they lower the relative performance of the proposed scheme to $88.1 \%$. As explained earlier, these performance characteristics of crypto are common to DES, but the difference is more emphasized.

Kxml's behavior is similar to those of SAXON with XSLT test cases. Its run length is only 10.8 bytecodes and the fraction of NF bytecode is $29.3 \%$. These factors explain the relatively low folding performance of $18.1 \%$ in PicoJava-II and $17.1 \%$ in the proposed scheme. In addition, we can observe that the largest fraction of folded bytecodes are in Group 3 (LV LV BG2). We can consider 


\begin{tabular}{|l||cccc|}
\hline \multicolumn{1}{|l||}{ Folding } & \multicolumn{4}{c|}{ SAXON with XSLTMark } \\
\cline { 2 - 5 } Scheme & chart & decoy & encrypt & trend \\
\hline \hline PJ2B & 68.6 & 70.3 & 81.9 & 79.5 \\
Proposed & 96.0 & 98.3 & 98.8 & 98.5 \\
\hline
\end{tabular}

\begin{tabular}{|l||ccc|}
\hline \multicolumn{1}{|l||}{ Folding } & \multicolumn{3}{c|}{ Other Benchmarks } \\
\cline { 2 - 4 } Scheme & ECM & DES & PNG \\
\hline \hline PJ2B & 80.6 & 67.1 & 77.2 \\
Proposed & 98.1 & 95.2 & 88.2 \\
\hline
\end{tabular}

\begin{tabular}{|l||cccc|}
\hline \multicolumn{1}{|l||}{ Folding } & \multicolumn{4}{c|}{ GrinderBench } \\
\cline { 2 - 5 } Scheme & chess & crypto & kxml & pngdecode \\
\hline \hline PJ2B & 77.3 & 66.1 & 66.5 & 66.2 \\
\hline Proposed & 99.7 & 88.1 & 94.2 & 74.0 \\
\hline
\end{tabular}

Table 4: Relative performance of two-bytecode version of PicoJava-II (PJ2B) and the proposed scheme normalized to PicoJava-II's four-bytecode folding scheme.

that kxml uses more conditional branches than arithmetic and logical operations. The fractions of OP and BG2 bytecode, $3.6 \%$ and $12.4 \%$, also support this idea.

It is expected that pngdecode exhibits the similar behavior as that of PNG. However, the difference among folding schemes is, again, more stressed. The relative performance of the proposed scheme is only $74.0 \%$, which is the worst figure among the benchmark programs used in this paper. The main contributor to this difference is obvious, the folding capability of Group 1. PicoJavaII's scheme folds $12.9 \%$ of the total bytecodes as Group 1, which is not possible in the proposed scheme. Another reason for the difference in the relative performance is SIPUSH bytecodes, which take $1.1 \%$ of the total number of bytecodes executed in pngdecode. This three-byte long bytecode is considered to be the source of differences in Groups 3 and 7.

The relative performance of $\mathrm{PJ} 2 \mathrm{~B}$ for GrinderBench ranges from $66.1 \%$ to $77.3 \%$, which is significantly lower than that of the proposed scheme, ranging from $74.0 \%$ to $99.7 \%$.

In principle, it is possible that opcodes form a foldable sequence but the folding of the sequence is not performed in the PicoJava-II. This is because, while the opcode of the last bytecode in the foldable sequence is present in the instruction buffer, its parameter is not. For example, the sequence of SIPUSH 0x100, ILOAD 4, IADD, ISTORE 5 is a Group 1 sequence and is eight byte long. While the opcode of the last bytecode ISTORE 5 is in the instruction buffer (and its folding type is decoded as MEM), its parameter (local variable index 5) is not. Therefore, this sequence cannot be folded as a Group 1. However, for the simulations in this paper, we did not see any instance of such "parameter overflow" in PicoJava-II, even for PNG which has the highest fraction of SIPUSH. 


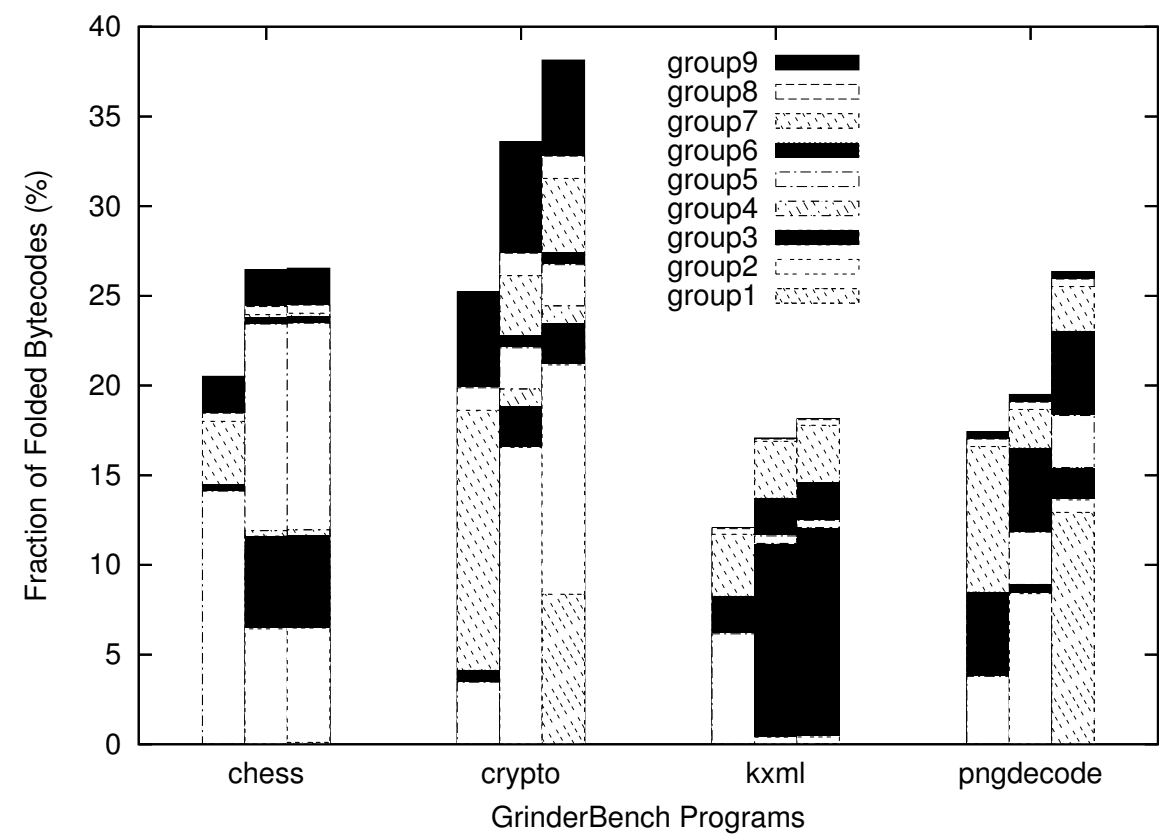

Figure 5: EEMBC benchmark programs. For each benchmark, results of two-bytecode version of PicoJava-II, proposed scheme, PicoJava-II's scheme are shown from left to right.

\section{Related Work}

ARM's Jazelle and Nazomi's Jstar are commercial products that incorporate hardware-translation in their JVMs. In this paper, the base design of the hardware-translation based JVM assumed the information published in ARM's white paper [3]. However, the ideas presented in this paper do not depend on the features specific to ARM or Nazomi's architectures and should be applicable to JVMs on most embedded RISC microprocessors.

PicoJava-II [8] directly executes Java bytecodes by the hardware. Since a pure JVM is not sufficient to build a real system, PicoJava's instruction set is extended for running applications written in "legacy" programming languages such as $\mathrm{C} / \mathrm{C}++$. Therefore, its design approach takes an opposite direction from the hardware-translation which tries to execute Java bytecodes by adding a small translation logic to the standard RISC type microprocessors. Our proposed instruction folding scheme is based on PicoJava-II.

Radhakrishnan et. al studied the microarchitecture of PicoJava-II and pointed out the instruction folding was the critical path of the processor pipeline [9]. To solve this issue, they proposed to move the instruction folding module from the decode stage of the pipeline to the instruction fill unit in the fetch stage. They also proposed to store the folded bytecodes in a dedicated cache (decoded bytecode cache) so that the folded bytecodes would be executed faster in the future.

Kim and Chang proposed a more aggressive folding mechanism which tried to find two or more foldable instruction sequences in which one breaks the sequence of the others [17]. The emphasis was placed on how to find such multiple sequences in the instruction stream and they did not work 
on how to fold each basic sequence (such as LV LV OP MEM). Moreover, since their scheme detects the foldable sequences by a state machine, it can be only used for ahead-of-time folding (and used later by storing it in a decoded bytecode cache as in [9]). Otherwise, if it is implemented by a combinational circuit, the size of the detection logic and instruction buffer will be larger than that for single sequence folding schemes.

Some embedded RISC processors can combine multiple operations into an instruction [18]. Such a combined instruction is converted to multiple operations before the decoding stage of the processor pipeline. This mechanism allows the RISC style instruction set (leading to a fixed instruction length and a simple decoding logic) and also a higher code density (reducing the instruction memory size). It is possible to pre-compile Java bytecodes into native instructions for a higher code density before deploying the program. However, one of the advantages of JVMs, the platform independence, will be lost in this case.

\section{Conclusions and Future Work}

In this paper, we proposed an instruction folding scheme for a hardware-translation based JVM. One of the sources of hardware complexity in the instruction folding in the PicoJava-II is the variable length of the bytecode format. The proposed scheme alleviated this problem by removing the SIPUSH which is the only three-byte long LV bytecode in the folding type classification of PicoJava-II. We also excluded the four-byte code sequence (LV LV OP MEM, Group 1 in PicoJavaII) so that the number of bytecodes decoded simultaneously is reduced from four to three. The proposed scheme achieved $74.0 \%$ to $99.7 \%$ of the PicoJava-II's scheme for seven benchmarks. In the image file analysis programs (PNG and pngdecode from GrinderBench), the effect of removing Group 1 sequence and SIPUSH bytecode were most significant. If we were to exclude these two programs, the worst relative performance of the proposed scheme would increase to $88.1 \%$.

Currently, a Group 1 sequence (LV LV OP MEM) is partially folded as a Group 2 sequence (LV LV OP) in the proposed scheme. However, if the local variable accessed in the first LV is not present in the local variable cache, we should have more chances of folding by discarding the first LV and handle the (partial) sequence of LV OP MEM as Group 4. Note that, LV loads a local variable while MEM stores to a local variable. Therefore, a MEM bytecode does not require the local variable to be present in the local variable cache and hence its access is hit as long as the index of the variable is with in the range of the local variable cache. A possible improvement for the proposed scheme is to look up the status of the local variable cache before folding. With this scheme, we can expect the folding ratio to be increased at the cost of local variable cache look-up.

In this paper, we evaluated the effectiveness of the proposed instruction scheme by the fraction of folded bytecodes. Actually, foldable bytecodes are only one of three types of codes executed on a JVM, other two are non-foldable (that include bytecodes that cannot be hardware-translated and are handled by software emulation) and native methods. We plan to develop a more complete model of a hardware-translation based JVM so that we can evaluate the performance by total execution time and power consumption of all three types of codes mentioned above.

Recently, ARM has combined the hardware translation with dynamic compilation technique [19]. The bytecode execution of a method takes advantages of hardware-translation during the first few invocations. When the method is identified to be executed frequently, it is complied dynamically. Such a hybrid execution model should also be considered for more realistic performance evaluation. 


\section{Acknowledgments}

This work is supported in part by The University of Aizu Competitive Research Funding. Yuichi Okuyama, from Computer Education Laboratory, The University of Aizu, contributed the area and delay estimation of the folding logic circuits in Section 3. The tools used in the estimation was provided by VLSI Design and Education Center (VDEC), the University of Tokyo in collaboration with Synopsys, Inc. The author would like to thank the comments from the anonymous reviewers and also the feedback from the audience at the Computing Frontiers 06 at which an earlier version of this paper was presented. They significantly improved the quality of this paper.

\section{References}

[1] Tim Lindholm and Frank Yellin, "The Java(TM) Virtual Machine Specification (2nd Edition)", Addison-Wesley Professional, 1999

[2] ORDER GRANTING MOTION FOR PARTIAL SUMMARY JUDGMENT OF NONINFRINGEMENT , Case No. C 02-02521-JF, UNITED STATES DISTRICT COURT, NORTHERN DISTRICT OF CALIFORNIA, SAN JOSE DIVISION, September 30, 2003.

[3] Steve Steel, "Accelerating to meet the challenges of embedded Java", White paper, ARM Limited, Nov 2001.

[4] "Jazelle - ARM Architecture Extensions for Java Applications", White paper, ARM Limited.

[5] "GrinderBench", http://www.grinderbench.com/.

[6] "the Embedded Microprocessor Benchmark Consortium", http: / /www . eembc . org / .

[7] Hitoshi Oi, "On the design of the local variable cache in a hardware translation-based java virtual machine", in Proceedings of the 2005 ACM SIGPLAN/SIGBED conference on Languages, compilers, and tools for embedded systems, pp87-94 June 2005.

[8] “PicoJava-II Microarchitecture Guide”, Sun Microsystems, March 1999.

[9] Ramesh Radhakrishnan, Deependra Talla and Lizy K. John, "Allowing for ILP in an embedded Java processor" in Proceedings of the 27th annual International Symposium on Computer architecture, Vancouver, British Columbia, Canada pp294-305, 2000.

[10] Kaffe.org, http://www.kaffe.org.

[11] "SAXON The XSLT and XQuery Processor", http://saxon. sourceforge. net/.

[12] "DataPower: XSLTMark XSLT Performance Benchmark", http://www. datapower.com/xmldev/xsltmark.html.

[13] "The Embedded CaffeineMark", Pendragon Software Corporation, 1997.

[14] "The Legion Of The Bouncy Castle", http: / /www. bouncycastle.org/

[15] "PNG SOFTWARE", http://www.sixlegs.com/software/png/. 
[16] "The USC-SIPI Image Database", http://sipi.usc.edu/database/.

[17] Austin Kim and Morris Chang, "Advanced POC Model-Based Java Instruction Folding Mechanism", in Proceedings of The 26th EUROMICRO Conference (EUROMICRO'00), Vol. I, No. 1 pp1332-1337, Sep. 2000.

[18] L. Nazhandali M. Minuth, B. Zhai, J. Olson, T. Austin and D. Blaauw, "A Second-Generation Sensor Network Processor with Application-Driven Optimizations and Out-of-Order Execution”, in Proceedings of CASES'05, pp249-256, September 2005.

[19] Chris Porthouse, "Jazelle for Execution Environments", Whitepaper, May 2005, ARM Limited. 